\title{
SELETIVIDADE DE HERBICIDAS LATIFOLICIDAS APLICADOS À MANDIOCA EM PÓS-EMERGÊNCIA
}

\author{
Dirceu Agostinetto ${ }^{1}$, Nilson Gilberto Fleck ${ }^{2}$, \\ Mauro Antonio Rizzardi ${ }^{3}$ e André Luis Thomas ${ }^{4}$
}

\author{
${ }^{1}$ Eng ${ }^{\circ}$. Agrônomo, MSc., Aluno do Programa de Pós-Graduação em Fitotecnia. UFRGS/Faculdade de Agronomia. Caixa Postal 776. \\ Porto Alegre, RS 91501-970 agostinetto@ig.com.br. \\ ${ }^{2}$ Eng $^{\circ}$. Agrônomo, Ph.D, Professor. UFRGS/Departamento de Plantas de Lavoura. \\ ${ }^{3}$ Eng $^{0}$. Agrônomo, MSc., Aluno do Programa de Pós-Graduação em Fitotecnia. UFRGS/Faculdade de Agronomia. \\ ${ }^{4}$ Eng$^{\circ}$. Agrônomo, MSc., Professor. UFRGS/Departamento de Plantas de Lavoura.
}

\section{RESUMO}

O principal problema de manejo na cultura da mandioca envolve a interferência exercida pelas plantas daninhas, as quais ocasionam elevadas reduções no rendimento de raízes. A inexistência de herbicidas de pós-emergência, torna o controle possível apenas pela aplicação de herbicidas de pré-emergência ou através de capinas manuais. O objetivo do experimento foi avaliar a seletividade de herbicidas com ação latifolicida aplicados em pós-emergência da cultura da mandioca. Para isso, foi conduzido um experimento na Faculdade de Agronomia da Universidade Federal do Rio Grande do Sul, em vasos com capacidade de 10 litros, nos quais foram plantadas quatro manivas. O delineamento experimental utilizado foi completamente casualizado, com cinco repetições. Os herbicidas utilizados foram: acifluorfen, bentazon, fomesafen, byspiribac, chlorimuron, imazethapyr, nicosulfuron e linuron. As variáveis avaliadas foram a fitotoxicidade dos herbicidas aos 4, 8, 14 e 28 dias após aplicação dos tratamentos (DAT) e a produção de fitomassa da parte aérea aos 14 e 28 DAT. O herbicida nicosulfuron apresentou seletividade à mandioca quando aplicado no estádio inicial de seu desenvolvimento, destacando-se dentre os tratamentos em produção de fitomassa, constituindo-se em opção promissora para controlar ervas dicotiledôneas nesta cultura.

Palavras-chave: controle químico, Manihot esculenta, plantas daninhas.

\section{ABSTRACT \\ Postemergence broadleaf herbicide selectivity in cassava}

The main management problem in the cassava crop in due to interference by weeds, where they bring about root yield reductions. Inexistence of selective postemergence herbicides, makes weed control only possible with preemergence herbicides or through hand hoeing. The objective of this research was to evaluate broadleaf herbicide selectivity when applied postemergence on cassava plants. An experiment was carried out at University of Rio Grande do Sul, Brazil, using 10-liter volume pots, where four cassava cuttings were planted. The experimental design used was a completely randomized one, with five replications. The tested herbicides were: acifluorfen, bentazon, fomesafen, byspiribac, chlorimuron, imazethapyr, nicosulfuron and linuron. The variables evaluated were herbicide injury at 4, 8, 14 and 28 days after treatment application (DAT), and shoot dry weight at 14 and 18 DAT. Nicosulfuron was selective for cassava when applied at an early growth stage, 
Dirceu Agostinetto et al.

significantly different from the other treatments as far as biomass production is concerned, constituting a promising option for selective broadleaf weed control in this crop.

Key-words: chemical control, Manihot esculenta, weeds.

\section{INTRODUÇÃO}

A cultura da mandioca (Manihot esculenta Crantz) caracteriza-se por apresentar ampla adaptação às condições edafoclimáticas e elevada importância alimentar para as populações de baixa renda. O Brasil é o segundo maior produtor de mandioca, contribuindo com cerca de $15 \%$ do total produzido no mundo (FAO, 1997). No Rio Grande do Sul a área cultivada no ano de 2000 alcançou 93 mil hectares, com produtividade média de 14,5 t/ha (IBGE/CEPAGRO, 2001). A mandioca pode ser utilizada na forma de farinha e de fécula para o consumo humano e como ração para animais (Dorretto, 1993); entretanto, no Rio Grande de Sul, aproximadamente $85 \%$ do total de raízes produzidas são usadas diretamente na alimentação animal, 14\% são utilizadas in natura na alimentação humana, e apenas $1 \%$ é industrializada na forma de farinha (Oyarzábal, 1995).

Os principais fatores limitantes ao cultivo da mandioca são: dificuldade de comercialização, impossibilidade de conservação do produto natural, política agrícola e infra-estrutura voltadas principalmente às culturas de exportação, interferência negativa de plantas daninhas e elevada demanda de mão-de-obra para cultivos em grandes áreas (Akobundu, 1980; Alcântara \& Ventorim, 1979).

A interferência exercida pelas plantas daninhas nos períodos iniciais de desenvolvimento da mandioca ocasiona reduções importantes no rendimento, sendo considerado o principal problema de manejo da cultura (Harper, 1974). Por apresentar crescimento inicial lento, a mandioca necessita de longo período de tempo para cobrir o solo (90 a 120 dias), oferecendo assim reduzido efeito supressor ao desenvolvimento de plantas daninhas. Nesta situação, o controle de plantas daninhas deve ser iniciado entre 15 e 30 dias após o plantio, mantendo-se a cultura livre de ervas até a formação completa do dossel (120 dias) (Doll \& Piedrahita, 1976). Por outro lado, foi observado que o desenvolvimento de plantas daninhas após 60 dias do plantio da mandioca não afetou negativamente o rendimento da cultura (Moura, 2000).

A inexistência de herbicidas pós-emergentes, recomendados para controle seletivo de plantas daninhas dicotiledôneas ocorrentes na cultura da mandioca, faz com que o controle seja normalmente realizado através de capinas manuais ou aplicação de herbicidas pré-emergentes. A capina é a principal causa de demanda de mão de obra na cultura, sendo responsável por cerca de $56 \%$ do total de tempo gasto (Pinstrup-Anderson \& Dias, 1973). A utilização de herbicidas de pré-emergência dificulta ao produtor selecionar o produto (ou dose) a ser utilizado e compromete a viabilidade econômica da prática de controle, visto que é difícil prever as espécies que podem emergir na área e também os seus níveis populacionais. Caso os herbicidas possam ser aplicados seletivamente em pós-emergência da cultura sem causar efeitos adversos, o controle de plantas daninhas seria facilitado. Desta forma, o objetivo do presente experimento foi avaliar a seletividade de herbicidas com ação latifolicida aplicados em pós-emergência na cultura da mandioca.

\section{MATERIAL E MÉTODOS}

O experimento foi realizado em telado na Faculdade de Agronomia da Universidade Federal do Rio Grande do Sul, no ano de 1999. Cada unidade experimental foi composta por um vaso com capacidade de $10 \mathrm{~L}$, preenchido com solo; nestes foram plantadas quatro manivas, possuindo em média, cinco gemas cada. O delineamento experimental utilizado foi completamente casualizado com cinco repetições. Os herbicidas pós-emergentes testados estão descritos na Tabela 1. Os produtos acifluorfen, bentazon e fomesafen são compostos que atuam por contato, enquanto os herbicidas

Tabela 1. Tratamentos herbicidas utilizados em pós-emergência em mandioca. Faculdade de Agronomia/UFRGS. Porto Alegre, RS, 1999.

\begin{tabular}{lccc}
\hline Nome comum & $\begin{array}{c}\text { Dose } \\
(\mathbf{g} / \mathbf{h a})\end{array}$ & Nome comercial & $\begin{array}{c}\text { Dose } \\
\text { (g ou L/ha) }\end{array}$ \\
\hline Acifluorfen & 255 & Blazer Sol & $1,5 \mathrm{~L}$ \\
Bentazon & 960 & Basagran 600 & $1,6 \mathrm{~L}$ \\
Fomesafen & 250 & Flex & $1,0 \mathrm{~L}$ \\
Byspiribac & 400 & Nominee $400 \mathrm{SC}$ & $1,0 \mathrm{~L}$ \\
Chlorimuron & 20 & Classic & $80,0 \mathrm{~g}$ \\
Imazethapyr & 100 & Pivot & $1,0 \mathrm{~L}$ \\
Nicosulfuron & 60 & Sanson 40 SC & $1,5 \mathrm{~L}$ \\
Linuron & 1575 & Afalon SC & $3,5 \mathrm{~L}$ \\
\hline
\end{tabular}


byspiribac-sodium, chlorimuron-ethyl, imazethapyr e nicosulfuron apresentam ação sistêmica. Além destes, aplicou-se também, em pós-emergência, como tratamento comparativo, o herbicida linuron, o qual está registrado para aplicação em pré-emergência da cultura. Na condição de pósemergência, o linuron apresenta ação basicamente de contato. Acrescentou-se um tratamento testemunha que não recebeu herbicida.

As aplicações herbicidas foram realizadas no horário compreendido entre $10 \mathrm{~h} 30$ e 11 horas, utilizando-se um pulverizador costal de precisão, pressurizado a $\mathrm{CO}_{2}$. Nele foram usados bicos de jato plano em leque da série 110.04, mantendo-se pressão constante de $1,5 \mathrm{kgf} / \mathrm{cm}^{2}$ e velocidade de deslocamento de $3,6 \mathrm{~km} / \mathrm{h}$, o que propiciou a aplicação de um volume de calda de $250 \mathrm{~L} / \mathrm{ha}$. Por ocasião da aplicação dos tratamentos as plantas de mandioca apresentavam estatura média de $6 \mathrm{~cm}$.

As variáveis avaliadas foram fitotoxicidade dos herbicidas aos 4, 8, 14 e 28 dias após a aplicação dos tratamentos (DAT) e produção de fitomassa aérea de mandioca aos 14 e 28 DAT. As avaliações de seletividade foram realizadas visualmente por dois pesquisadores, sendo atribuídos valores em escala percentual, variando de $0 \%$ (ausência de dano) a $100 \%$ (plantas mortas). Para determinação da fitomassa seca, as plantas foram colocadas em estufa com circulação de ar quente a $60{ }^{\circ} \mathrm{C}$ por período de 72 horas. Os resultados obtidos foram submetidos a análise de variância e a comparação de médias foi realizada pelo teste de Duncan ao nível de $5 \%$ de probabilidade. Os dados de fitotoxicidade foram previamente transformados por arco seno da raiz quadrada de $x+100$.

\section{RESULTADOS E DISCUSSÃO}

Na primeira avaliação de fitotoxicidade, os herbicidas com ação de contato apresentaram injúria à cultura, superior aos herbicidas de ação sistêmica e ao tratamento padrão (Tabela 2). O herbicida bentazon foi exceção, provocando menor injúria dentro de seu grupo e, também, comparativamente ao linuron. Na avaliação realizada aos $8 \mathrm{DAT}$, verificou-se que os herbicidas, exceto nicosulfuron, apresentaram elevada ação fitotóxica, variável entre 54 e $82 \%$. Na terceira avaliação, o herbicida nicosulfuron continuou a apresentar menor efeito fitotóxico. De modo geral, os demais herbicidas não diferiram entre si, apresentando respostas variáveis entre 34 e $50 \%$ de injúria, (exceto linuron que atingiu nível de $84 \%$ ). Na última avaliação, as maiores percentagens de injúria foram observadas para os tratamentos de chlorimuron e imazethapyr, os quais não diferiram do padrão. Contudo, nesta avaliação observou-se acentuada recuperação das plantas de mandioca, especialmente para os produtos com ação de contato em relação às avaliações realizadas até os 14 dias, sendo que estes não diferiram do herbicida nicosulfuron e da testemunha.

Os sintomas aparentes para os herbicidas com ação de contato foram representados por "bronzeamento" folhar, clorose e áreas que progrediram para necrose. Ainda, para linuron, houve clorose internervural mais intensa e amarelecimento das margens das folhas, sintomas ocorrentes especialmente nas folhas mais velhas. Para produtos com ação sistêmica, os sintomas gerais foram inibição do crescimento, o que ocorreu com mais intensidade 1 a 2 semanas após as aplicações. Para estes herbicidas também se constatou algu-

Tabela 2. Fitotoxicidade de herbicidas aplicados em pós-emergência na cultura da mandioca. Faculdade de Agronomia/ UFRGS. Porto Alegre, RS, 1999.

\begin{tabular}{|c|c|c|c|c|c|}
\hline \multirow{2}{*}{ Herbicida } & \multirow{2}{*}{$\begin{array}{c}\text { Dose } \\
\text { (g/ha) }\end{array}$} & \multicolumn{4}{|c|}{ Fitotoxicidade $(\%)^{2}$} \\
\hline & & $4 \mathrm{DAT}^{1}$ & $8 \mathrm{DAT}$ & $14 \mathrm{DAT}$ & $28 \mathrm{DAT}$ \\
\hline Acifluorfen & 255 & $77 \mathrm{a}^{3}$ & $75 \mathrm{~b}$ & $42 b c$ & $0 \mathrm{~b}$ \\
\hline Bentazon & 960 & $52 \mathrm{~d}$ & $66 c$ & $43 \mathrm{bc}$ & $1 \mathrm{~b}$ \\
\hline Fom esafen & 250 & $71 \mathrm{~b}$ & $66 c$ & $44 \mathrm{bc}$ & $1 \mathrm{~b}$ \\
\hline Byspiribac & 400 & $12 \mathrm{e}$ & $60 \mathrm{~cd}$ & $48 \mathrm{~b}$ & $1 \mathrm{~b}$ \\
\hline Chlorimuron & 20 & $11 \mathrm{e}$ & $54 \mathrm{~d}$ & $50 \mathrm{~b}$ & $19 \mathrm{a}$ \\
\hline Imazetaphyr & 100 & $8 \mathrm{e}$ & $63 c$ & $34 \mathrm{c}$ & $15 \mathrm{a}$ \\
\hline Nicosulfuron & 60 & $9 \mathrm{e}$ & $5 \mathrm{e}$ & $5 \mathrm{~d}$ & $1 \mathrm{~b}$ \\
\hline Linuron & 1575 & $62 \mathrm{c}$ & $83 \mathrm{a}$ & $84 \mathrm{a}$ & $13 \mathrm{a}$ \\
\hline Testemunha & - & $0 \mathrm{f}$ & $0 \mathrm{f}$ & $0 \mathrm{e}$ & $0 \mathrm{~b}$ \\
\hline C.V. $(\%)$ & & 8,0 & 7,1 & 14,3 & 64,5 \\
\hline
\end{tabular}

${ }^{1}$ Refere-se ao número de dias após a aplicação dos tratamentos.

${ }^{2} 0 \%=$ ausência de dano; $100 \%=$ plantas mortas.

${ }^{3}$ Médias seguidas por mesma letra nas colunas, não diferem entre si pelo teste de Duncan ao nível de 5\% de probabilidade. 
ma clorose folhar alguns dias após tratamento, especialmente em regiões meristemáticas.

As diferenças verificadas entre tratamentos nas primeiras avaliações deveram-se basicamente ao modo de ação dos herbicidas. Para herbicidas com ação de contato (acifluorfen, bentazon e fomesafen) que atuam próximo ao local de absorção (Ahrens, 1994), os danos surgiram imediatamente após a aplicação e permaneceram elevados nos 8 primeiros dias; a partir daí reduziram-se acentuadamente. Para herbicidas sistêmicos (byspiribac, chlorimuron e imazethapyr), que são translocados até o local de ação (Ahrens, 1994), os danos aumentaram até os 8 DAT e permaneceram altos durante os primeiros 14 dias, para só então sofrer redução.

A maior produção de fitomassa aos 14 DAT foi observada para o tratamento nicosulfuron, de valor similar à testemunha e superior aos demais herbicidas, os quais em geral não diferiram entre si (Tabela 3). Aos 28 DAT, nicosulfuron ainda apresentou maior produção de fitomassa que chlorimuron e linuron; entretanto, já não diferiu dos demais herbicidas. Estes resultados mostram recuperação acentuada dos danos ao crescimento aéreo da mandioca entre 2 e 4 semanas após as aplicações herbicidas, exceto para linuron.

$\mathrm{Na}$ literatura são encontrados trabalhos de pesquisa referentes ao controle químico de plantas daninhas na cultura da mandioca (Akobundu, 1980; Alcântara \& Venturim, 1979; Silva et al., 1979; Unamma et al., 1986). No entanto, a seletividade de herbicidas latifolicidas pós-emergentes ainda é pouco explorada, não se encontrando trabalhos de pesquisa nesta linha. Além disso, o elevado custo econômico da capina manual em grandes áreas, aliado ao período total de prevenção da interferência das plantas daninhas na cultura da mandioca, que é de aproximadamente 60 dias (Moura, 2000), demonstram a necessidade de estudos adicionais que identifiquem produtos, doses e épocas de aplicação de herbicidas pós-emergentes para o controle seletivo de plantas daninhas dicotiledôneas na cultura da mandioca.

Em geral, os herbicidas com ação de contato apresentaram elevado impacto inicial de injúria às plantas de mandioca, mas também mostraram rápida recuperação dos danos. Inicialmente, para esses produtos, acicluorfen provocou a maior fitotoxicidade e bentazon a menor. Para o grupo de compostos com ação sistêmica, o impacto negativo de injúria surgiu mais lentamente, o mesmo comportamento ocorrendo com a recuperação das plantas de mandioca. No caso dos produtos com este modo de ação, nicosulfuron mostrou nível satisfatório de seletividade à mandioca, de modo a se indicar sua recomendação potencial para a cultura. No entanto, os resultados obtidos neste experimento oferecem informações preliminares que deverão ser confirmados por trabalhos conduzidos em condições de campo.

\section{CONCLUSÕES}

Dentre os herbicidas testados, nicosulfuron é o que apresenta maior grau de seletividade à mandioca desde 2 semanas após a aplicação e por isso se mostra mais promissor do que os demais como opção para controle seletivo de ervas dicotiledôneas nesta cultura.

Os herbicidas acifluorfen, bentazon, fomesafen, byspiribac e imazethapyr apresentam grau de injúria elevada nas primeiras 2 semanas após aplicação, mas as plantas de mandioca se recuperam do dano após 4 semanas da aplicação, de modo que nesta época eles se posicionaram em equivalência ao nicosulfuron quanto à seletividade.

Os herbicidas chlorimuron e linuron, em aplicação de pós-emergência, são os produtos que demonstram menor seletividade à mandioca e que mostram baixo potencial para uso nesta cultura, na modalidade em que foram aplicados.

Tabela 3. Produção de fitomassa aérea pela cultura da mandioca em resposta a herbicidas pós-emergentes. Faculdade de Agronomia/UFRGS, Porto Alegre, RS, 1999.

\begin{tabular}{|c|c|c|c|c|c|}
\hline \multirow[b]{2}{*}{ Herbicida } & \multirow{2}{*}{$\begin{array}{l}\text { Dose } \\
\text { (g/ha) }\end{array}$} & \multicolumn{4}{|c|}{ Fitomassa (g/vaso) } \\
\hline & & $14 \mathrm{DAT}^{1}$ & $\begin{array}{c}\text { Valor relativo } \\
(\%)^{2}\end{array}$ & 28 DAT & $\begin{array}{c}\text { Valor relativo } \\
(\%)\end{array}$ \\
\hline Acifluorfen & 255 & $1,98 \mathrm{~b}^{3}$ & 47,1 & $6,16 \mathrm{ab}$ & 90,1 \\
\hline Bentazon & 960 & $1,89 \mathrm{bc}$ & 45,0 & $6,34 \mathrm{ab}$ & 92,7 \\
\hline Fomesafen & 250 & $1,99 \mathrm{~b}$ & 47,4 & $5,46 \mathrm{abc}$ & 79,8 \\
\hline Byspiribac & 400 & $1,95 \mathrm{~b}$ & 46,4 & $5,96 \mathrm{ab}$ & 87,1 \\
\hline Chlorimuron & 20 & $1,39 \mathrm{bc}$ & 33,1 & $4,18 \mathrm{bc}$ & 61,1 \\
\hline Imazethapyr & 100 & $2,26 \mathrm{~b}$ & 53,8 & $5,76 \mathrm{ab}$ & 84,2 \\
\hline Nicosulfuron & 60 & $3,35 \mathrm{a}$ & 79,8 & $6,69 \mathrm{a}$ & 97,8 \\
\hline Linuron & 1575 & $0,86 \mathrm{c}$ & 20,5 & $3,37 \mathrm{c}$ & 49,3 \\
\hline Testemunha & - & $4,20 \mathrm{a}$ & 100,0 & $6,84 \mathrm{a}$ & 100,0 \\
\hline C.V. $(\%)$ & & 35,2 & & 28,4 & \\
\hline
\end{tabular}

${ }^{1}$ Refere-se ao número de dias após a aplicação dos tratamentos.

${ }^{2}$ Comparativo à testemunha.

${ }^{3}$ Médias seguidas por mesma letra nas colunas, não diferem entre si pelo teste de Duncan ao nível de 5\% de probabilidade. 


\section{LITERATURA CITADA}

AHRENS, W.H., ed. Herbicide handbook. Champaign: Weed Science Society of America, 1994. 352p.

AKOBUNDU, I.O. Weed control in cassava cultivation in the subhumid tropics. Tropical Pest Management, v.26, n.4, p.420-426, 1980.

ALCÂNTARA, E.N. de; VENTORIM, N. Controle de ervas daninhas na cultura da mandioca. Informe Agropecuário, v.5, n.59-60, p.50-53, 1979.

DOLL, J.D.; PIEDRAHITA, W.C. Metodos de control de malezas em yuca. Cali: CIAT, 1976.12p. (CIAT. Séries EE-21).

DORRETTO, M. Distribuição da cultura da mandioca no Paraná nos anos 80. Londrina: IAPAR, 1993. 19p. (Informe Agropecuário, 102).

FAO. FAO yearbook production, v. 15, 1997.

HARPER, R.S. Chemical weed control in cassava using paraquat. PANS, v.20, n.2, p.285-289, 1974.

IBGE/CEPAGRO. Levantamento sistemático da produção agrícola. Disponível na internet. http://www.ibge.net/ ibge/estatística/indicadores/agropecuária, em 15 de março de 2001.

MOURA, G.M. Interferência de plantas daninhas na cultura da mandioca (Manihot esculenta) no Estado do Acre. Planta Daninha, v.18, n.3, p.451-456, 2000.

OYARZÁBAL, G.N. Aproveitamento integral da mandioca no Rio Grande do Sul: rações à base de mandioca. Porto Alegre: EMATER, RS, 1995. 64p.

PINSTRUP-ANDERSON, P.; DIAZ, R.O. Present and potential labour absorption in cassava production in Colombia. In: THIRD INTERNATIONAL SYMPOSIUM ON TROPICAL ROOT CROPS, 3. Ibadan, 1973. Proceedings... Ibadan, 1973. p.54-57.

SILVA, A.A. da; SILVA, J.F. da; BEGAZO, J.C.E.; CONDE, A.R. Controle químico de plantas daninhas na cultura da mandioca (Manihot esculenta Crantz). Cariacica: EMCAPA, 1979. 26p. (Boletim Técnico, 1).

UNAMMA, R.P.A.; ENE, L.S.O.; ODURUKWE, O.; ENYINNIA, T. Integrated weed management for cassava intercropped with maize. Weed Research, v.26, n.1, p.9-17, 1986. 
